\title{
Addressing Underserved Populations and Unmet Needs
}

\author{
By Eric Hollander, MD
}

In this issue of CNS Spectrums we examine some underserved populations who continue to have real unmet needs. For example, patients in forensic and correctional settings have serious psychopathology resulting in substantial morbidity and mortality, and yet human subjects concerns have led them to be very much understudied. Likewise, patients with treatment-resistant obsessive-compulsive disorder (OCD) are quite common, and yet, compared with other disorders such as schizophrenia, few resources have been devoted to addressing their substantial functional disability. Finally, cognitive dysfunction in the elderly is an enormous problem, yet little work has addressed the role of gonadal dysfunction, or male menopause, in specific cognitive deficits of the elderly.

Donald W. Black, MD, and colleagues conducted a pilot study of the Systems Training for Emotional Predictability and Problem Solving, a cognitive-behavioral group treatment for individuals with borderline personality disorder (BPD), in incarcerated women offenders. There were significant improvements in BPDrelated symptoms, negative affectivity, and depression, and overall improvement in BPDrelated symptoms. Effect sizes for the efficacy measures were moderate to large, and there was excellent adherence. Participants achieved "real-world" benefits. Clearly, there is a need for evidence-based approaches to the treatment of severe Axis II personality disorders. Furthermore, the United States population in prison continues to grow at a high rate. Since impulsive aggression associated with BPD is a serious management problem in forensic settings, such research is sorely needed. However, there are special challenges to conducting human subject research in such correctional settings. The authors should be commended for conducting such research in this difficult-tostudy but underserved population.

Michael J. Serby, MD, and colleagues examined the relationship between testosterone (endogenous testosterone and the effects of androgen depletion and replacement) and various cognitive domains (including memory and visuospatial function) in elderly men. Of interest, in certain conditions (ie, hypogonadism, cognitive impairment) testosterone levels may be significantly associated with memory and visuospatial-function deficits, but not deficits of other cognitive functions. Clearly, studies with larger sample sizes and more sensitive cognitive measures are indicated as questions remain about the potential role of testosterone as a cognitive enhancer. Again, another unexplored realm of men's health involves the role of gonadal function and cognitive deficits in the elderly.

George M. Simpson, MD, and colleagues evaluated the long-term efficacy and tolerability

Dr. Hollander is the editor of this journal, Esther and Joseph Klingenstein Professor and Chairman of Psychiatry at the Mount Sinai School of Medicine, and director of the Seaver and New York Autism Center of Excellence in New York City. 
of treatment with ziprasidone following a switch from prior 6-week trials of antipsychotics (conventional, olanzapine, or risperidone) in patients with schizophrenia or schizoaffective disorder in three open-label, flexible-dose, 1-year extension studies. Patients showed significant improvement in Positive and Negative Syndrome Scale total scores, Positive and Negative Syndrome Scale negative scores, and Clinical Global Impression of severity score. Ziprasidone was well tolerated; and patients demonstrated significant improvement in metabolic parameters and in movement disorder assessments. Insomnia and somnolence occurred in $>10 \%$ of subjects. Patients with serious mental illness need various treatment options in order to optimize the risk-benefit ratio, and to personalize the treatment to the individual.

Rupert Conrad, MD, and colleagues report the case of a young man with a large calcification in the right thalamus, which was first diagnosed at 9 years of age. Case history reveals specific eating rituals and other obsessive-compulsive personality traits in the ehildhood and adolescence. After a critical life event the patient developed anorexia nervosa. Involvement of specific thalamic structures, such as the dorsomedial nucleus, appear to be involved in the development of anorexia nervosa. Furthermore, the treatment of the patient by a combined psychotherapeutic and pharmacotherapeutic approach with olanzapine, which can induce weight gain by an increase of leptin levels, is described. Sometimes, unique cases that involve specific lesions associated with the development of psychiatric sequela can be informative in developing models of underlying brain circuitry.

CNS Spectrums will continue to examine underserved populations who have real unmet needs, and continue to suggest new lines of treatment based on our understanding of their underlying pathophysiology and functional deficits. CNS 